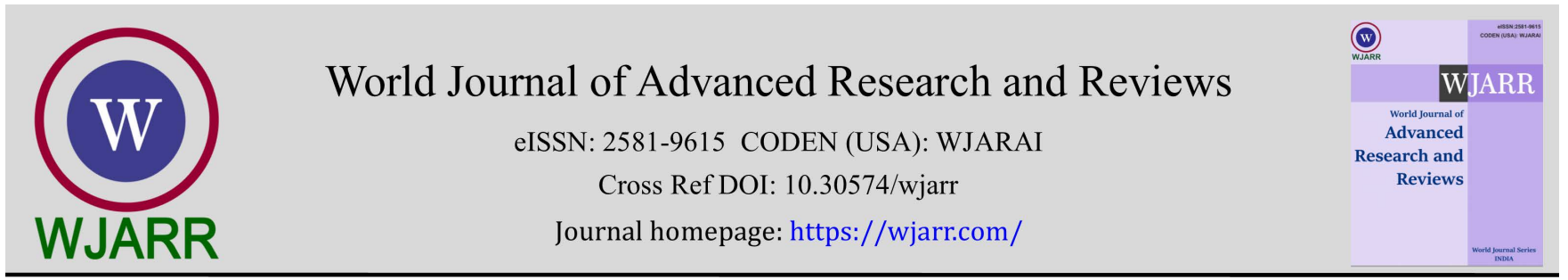

(RESEARCH ARTiClE)

Check for updates

\title{
Impact of Covid-19 pandemic on the clinical profile of patients in Neurophysiology clinic
}

\author{
Mamdouh H. Kalakatawi ${ }^{1}$ and Nusrat Aziz ${ }^{1,2, *}$ \\ ${ }^{1}$ Department of Neurology, Al Noor Specialists Hospital, Makkah, Kingdom of Saudi Arabia. \\ ${ }^{2}$ Department of Physiology, Faculty of Medicine, Umm Al-Qura University, Makkah 24382, Kingdom of Saudi Arabia.
}

World Journal of Advanced Research and Reviews, 2021, 09(03), 319-325

Publication history: Received on 20 February 2021; revised on 22 March 2021; accepted on 25 March 2021

Article DOI: https://doi.org/10.30574/wjarr.2021.9.3.0120

\begin{abstract}
Clinical neurophysiological testing is an important integral part of neurological diagnosis and management. During Covid-19 pandemic non-emergency clinics have suffered adversely. WHO declared Covid-19 pandemic in March 2020.

Clinical records from March 2019 to March 2021 were analyzed, for impact of pandemic on profile of 924 patients referred for nerve conduction studies (NCS).

It was observed that percentage of referrals showing normal NCS decreased from 48.64\% to 28.45\%, Carpal tunnel syndrome (CTS) cases - decreased from $60.45 \%$ to $46.58 \%$ of the total abnormal cases, mild CTS cases - decreased from $36.11 \%$ of to $26.2 \%$ of total CTS patients, traumatic nerve injuries -increased from $6.96 \%$ before Covid-19 to $18.02 \%$ during the pandemic and the radiculopathies diagnosed - increased from $7.8 \%$ to $11.8 \%$.
\end{abstract}

Pandemics are difficult situations and these findings prompt us to question "Is the prolonged stay at home, and work from home damaging our spine and increasing radiculopathies?" "Are we inadvertently missing the milder cases?" "Is the pandemic stress taking its toll in increased percentage of traumatic nerve injuries?"

There is a urgent need to study more about this effect. This will help us to be better prepared for such pandemics in future, and bring about necessary changes in our healthcare system.

Keywords: Clinical neurophysiology; Nerve conduction study; Covid-19; Pandemic

\section{Introduction}

Clinical neurophysiology is an important integral part of neurology department. It deals with diagnosis of central and peripheral neurological disorders using electrical potential recordings and hence has been called as Electrodiagnostic test (EDX). Neurophysiological testing provides valuable information to support the history and clinical examinations to reach a final diagnosis. Using electricity for studying the nerves and muscles first started with Luigi Galvani in 1791 by his experiments on frog muscle nerve preparation showing that nerves and muscles had intrinsic electrical activity which he described in his work Commentarius [1]. 50 years later German physiologist Du Bois-Raymond could record an action potential and has been called as the father of electrophysiology [2] and by 1850 the scientist von Helmhotz successfully made a device for measuring conduction velocity of nerves [1] The first Electromyography (EMG) machine was built by Jasper in 1942 [3]. Since then, neurophysiological studies had played an important and indispensable role in diagnosis of Neurological disorders. Neuromuscular disorders, that is abnormalities of nerves, muscles and the

\footnotetext{
${ }^{*}$ Corresponding author: Dr. Nusrat Aziz

Department of Physiology, Faculty of Medicine, Umm Al-Qura University, Makkah 24382, Kingdom of Saudi Arabia.

Copyright $(2021$ Author(s) retain the copyright of this article. This article is published under the terms of the Creative Commons Attribution Liscense 4.0.
} 
neuromuscular junction can be diagnosed by technique of electromyography (EMG) and nerve conduction studies (NCS). In this article we analyze the clinical profile of patients referred for nerve conduction studies in neurophysiology clinic of Al Noor Specialist Hospital, a tertiary care hospital in Makkah in Saudi Arabia. and its alteration in consequence of Covid-19 pandemic.

Pandemics are difficult circumstances with a great impact on our physical and mental health. World Health Organization (WHO) had declared on 11 march 2020 the state of pandemic for Covid-19 virus. [4]. Covid -19 virus spreads by aerosol and by contact with infected persons or surfaces. Pandemic is a period of increased alertness and vigilance. WHO has declared 3.4\% mortality rate by March 2020 [5]. There has been heightened awareness and caution exercised by the governments and the health sector to control and limit the pandemic and its effects. The number of new cases in Saudi Arabia reported had declined below 400 during the month of October 2020 and had remained low since then [6]. Though the pandemic has largely been controlled in Saudi Arabia by efficient timely interventions but still the stress and fear prevail. This stress and fear of morbidity and mortality has adversely affected the clinics. We attempt to evaluate the consequences of the pandemic on the profile of patients coming to the neurophysiological clinic for nerve conduction studies (NCS) in a tertiary care hospital and compare with that of pre-covid 19 period.

Nerve conduction studies (NCS) help in detecting the abnormalities of peripheral nervous system. By NCS one can detect lesion in spinal cord, spinal root, plexus, peripheral nerve, neuromuscular junction (NMJ) [7]. These studies help in localizing the disease, ascertaining its severity, defining the phenotype of type of disease and its possible pathophysiology. NCS results can even catch subclinical disease and thus lead to better prognosis.

The technique of NCS involves measuring electrical activity over the nerves and muscles using surface recording electrodes after stimulating with minute electrical current using stimulating electrodes over the course of the nerve. Study of sensory nerve action potential (SNAP) and compound muscle action potential (CMAP) are the basic procedures applied for the diagnosis. This is supported by special techniques such as F-Wave study for proximal part of peripheral nerves, $\mathrm{H}$ Wave study for reflex arch pathway, repeated nerve stimulation (RNS) for Myasthenia Gravis and inching technique for conductional blocks.

\section{Study design}

A cross-sectional study was done from the patient records in the Neurophysiology Clinic from 2019 to 2021 and analyzed. This study was conducted in Al Noor Specialist hospital in Makkah in the western province of Saudi Arabia. Al Noor specialist hospital is a tertiary care hospital. The Neurophysiology clinic receives patients referred from other departments in the same hospital and also from primary and secondary care hospitals of Makkah and regions around it. In view of pandemic the Neurophysiology outpatient consultation for elective cases was closed from March 2020 to October 2020. The clinic was restarted in October 2020. Guidelines provided for safety of physicians and the technical staff were followed including face masks, Personal protective equipment, cleaning and sterilization with distancing measures in outpatient clinics and waiting rooms were followed $[8,9,10]$ and utmost care was taken to protect the neurophysiology clinic staff. Precautionary measures such as mandatory use of masks, gloves and other PPE while keeping 1 metre distancing between patients in Outpatient department were taken. The patients showing respiratory symptoms or increase temperature were checked at the entrance and referred to the emergency department for testing for Covid-19 infection. The patient records from march 2019 to march 2020 were taken as pre-Covid. The data of patients from October 2020 to March 2021 as the data during the pandemic. The period from March 2020 to March 2021 the clinic was closed and no elective neurophysiological testing was done. The data of 924 individuals referred to Neurophysiology clinic were analyzed.

\subsection{Statistics}

Statistical package of Social Sciences (SPSS 20) was used to analyze the data. Descriptive studies were done to find out the frequencies and percentages of different conditions. Charts and bar diagrams were prepared for a clear understanding of the results.

\section{Results}

A record of data was collected of patients referred to the Neurophysiology clinic from march 2019 to march 2021 was analyzed. Total of 924 subjects were referred to the clinic. Of these 404 subjects showed normal results and 520 individuals showing abnormal nerve conduction studies. The abnormal results were divided according to the diagnosis made and the data analyzed. The pre-Covid (during the period from March 2019 to March 2020) data showed turnover of 699 patients of which showed 359 (51.36\%) patients showing abnormal NCS and 340 (48.64\%) had normal NCS. The 
different diagnosis made in 359 patients with abnormal NCS were Carpal tunnel syndrome (CTS) in 217 patients (60.45\%), peripheral neuropathy56 (15.6\%), radiculopathies 28(7.8\%), nerve injuries 25 ( 6.96\%), Myasthenia Gravis $5(0.56 \%)$, Guillain Barre Syndrome $3(0.2 \%)$, mononeuropathy including ulnar, radial, peroneal, tibial and facial nerves $23(6,4 \%)$ and plexopathy including brachial and lumbosacral plexopathy were $2(0.56 \%)$. The details of this are given in Table: 1 given below.

Table 1 Profile of patients attending Neurophysiology clinic before the Covid-19 pandemic

\begin{tabular}{|c|c|c|c|c|c|}
\hline & & \multicolumn{2}{|l|}{ Gender } & \multirow[t]{2}{*}{ Total } & \multirow{2}{*}{$\begin{array}{l}\% \text { of total } \\
\text { abnormal } \\
\text { conditions }\end{array}$} \\
\hline & & Count in males & Count in females & & \\
\hline \multirow{12}{*}{$\begin{array}{l}\text { Diagnosis } \\
\text { before Covid } \\
19 \text { Pandemic }\end{array}$} & Peripheral neuropathy & 35 & 21 & 56 & $15.6 \%$ \\
\hline & Carpal tunnel syndrome & 55 & 162 & 217 & $60.45 \%$ \\
\hline & Radiculopathy & 19 & 9 & 28 & $7.8 \%$ \\
\hline & Nerve injury & 19 & 6 & 25 & $6.96 \%$ \\
\hline & Myesthenia gravis & 2 & 3 & 5 & $0.56 \%$ \\
\hline & Guillian barre syndrome & 1 & 2 & 3 & $0.2 \%$ \\
\hline & Ulnar neuropathy & 6 & 2 & 8 & $2.2 \%$ \\
\hline & Radial neuropathy & 1 & 3 & 4 & $1.22 \%$ \\
\hline & Peroneal neuropathy & 6 & 1 & 7 & $1.67 \%$ \\
\hline & Tibial Neuropathy & 2 & 0 & 2 & $0.56 \%$ \\
\hline & Facial neuropathy & 1 & 1 & 2 & $0.56 \%$ \\
\hline & Plexopathy & 2 & 0 & 2 & $0.56 \%$ \\
\hline \multicolumn{2}{|l|}{ Total } & 149 & 210 & 359 & $100 \%$ \\
\hline
\end{tabular}

The Neurophysiology clinic was closed during March 2020 to October 2020 following the protocol for pandemic management. The patient turnover during the Covid -19 pandemic from October 2020 to March 2021 was lesser with only 225 patients. 64 of them had normal NCS (28.45\%) with $161(71.56 \%)$ patients having abnormal NCS. Of the 161 abnormal NCS the diagnosis made and number and percentage of total abnormal were CTS -75 (46.58\%), peripheral neuropathy 26 (16.1\%), radiculopathy 19 (11.8\%), nerve injuries (18.02\%) mononeuropathy including ulnar, radial, peroneal and tibial nerves were $11(1.2 \%)$ and plexopathy was $1(0.6 \%)$. The details of it are given in Table:2 below.

Table 2 Profile of patients attending Neurophysiology clinic before the Covid-19 pandemic

\begin{tabular}{|c|c|c|c|c|}
\hline & & \multicolumn{2}{|l|}{ Gender } & \multirow[t]{2}{*}{ Total } \\
\hline & & Count in males & Count in females & \\
\hline \multirow{9}{*}{$\begin{array}{l}\text { Diagnosis during } \\
\text { covid19 } \\
\text { pandemic }\end{array}$} & Peripheral neuropathy & 11 & 15 & 26 \\
\hline & Carpal tunnel syndrome & 17 & 58 & 75 \\
\hline & Radiculopathy & 10 & 9 & 19 \\
\hline & Nerve injury & 22 & 7 & 29 \\
\hline & Ulnar neuropathy & 2 & 1 & 3 \\
\hline & Radial neuropathy & 0 & 3 & 3 \\
\hline & Peroneal neuropathy & 3 & 1 & 4 \\
\hline & Tibial neuropathy & 1 & 0 & 1 \\
\hline & Plexopathy & 1 & 0 & 1 \\
\hline \multicolumn{2}{|l|}{ Total } & 67 & 94 & 161 \\
\hline
\end{tabular}


The percentages of different neurological disorders diagnosed before and during the Covid-19 pandemic are shown below in Fig: 1 and Fig: 2 respectively.

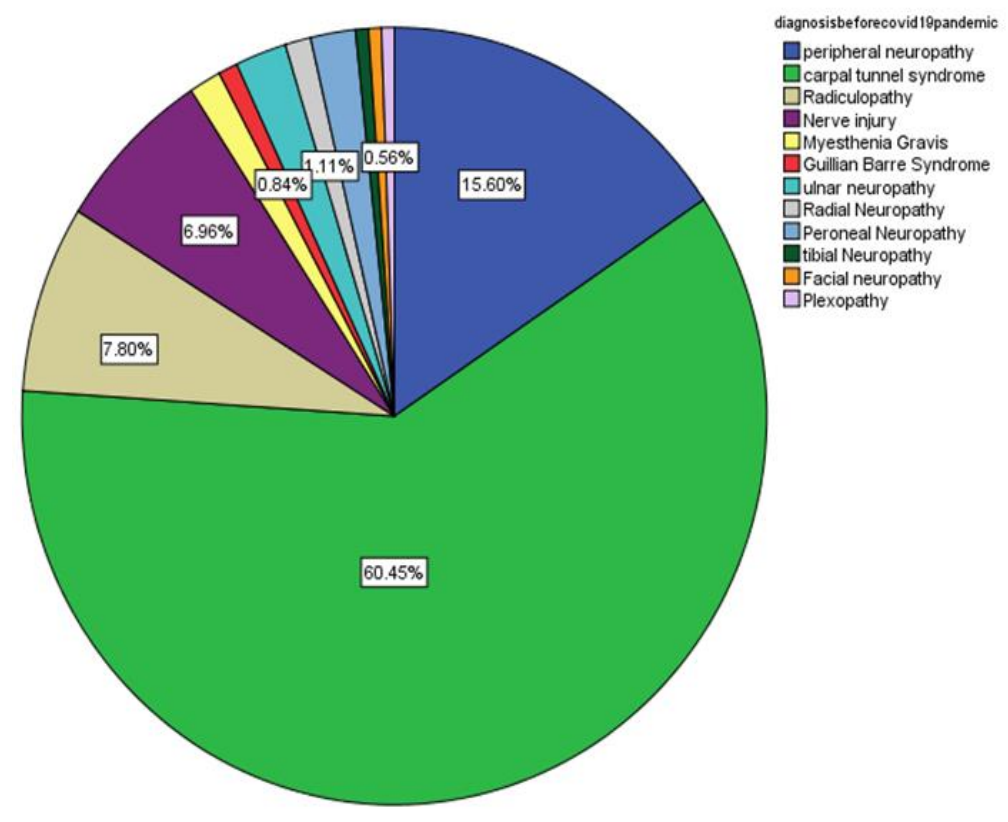

Figure 1 Percentages of Neurological disorders diagnosed by neurophysiological findings before Covid-19 pandemic.

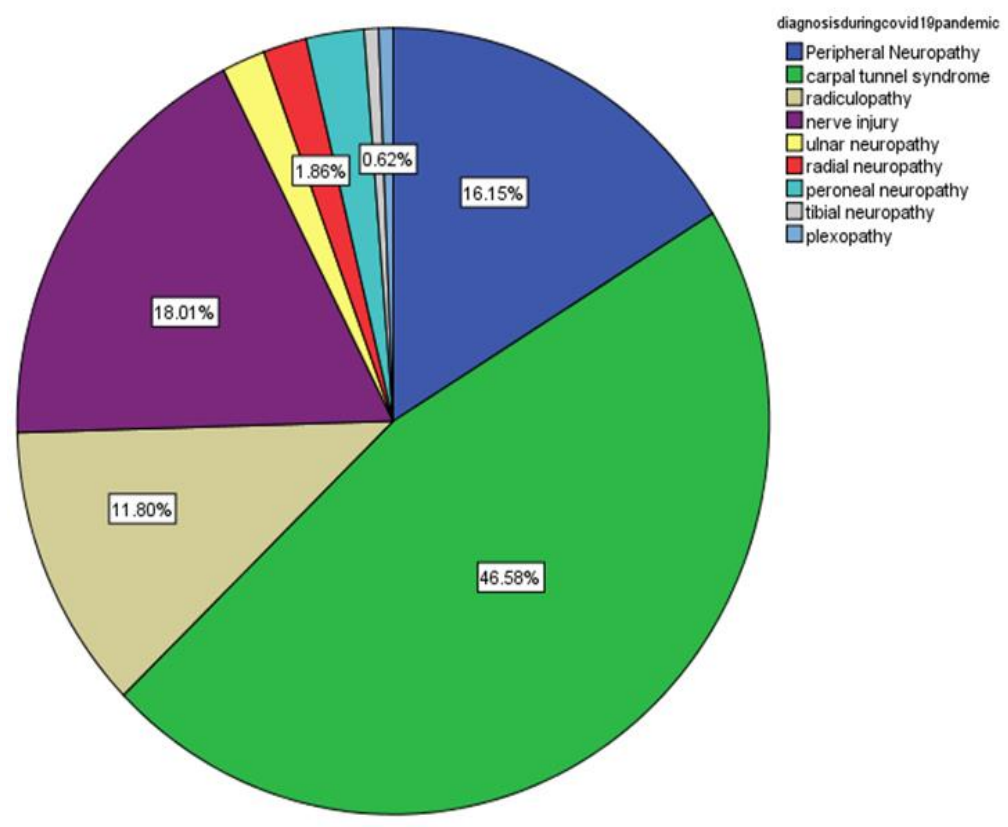

Figure 2 Percentages of Neurological disorders diagnosed by neurophysiological findings during the Covid-19 pandemic

The distribution of neurological cases diagnosed according to gender for Pre-Covid and during pandemic are shown in Fig:3 and Fig:4 respectively. 


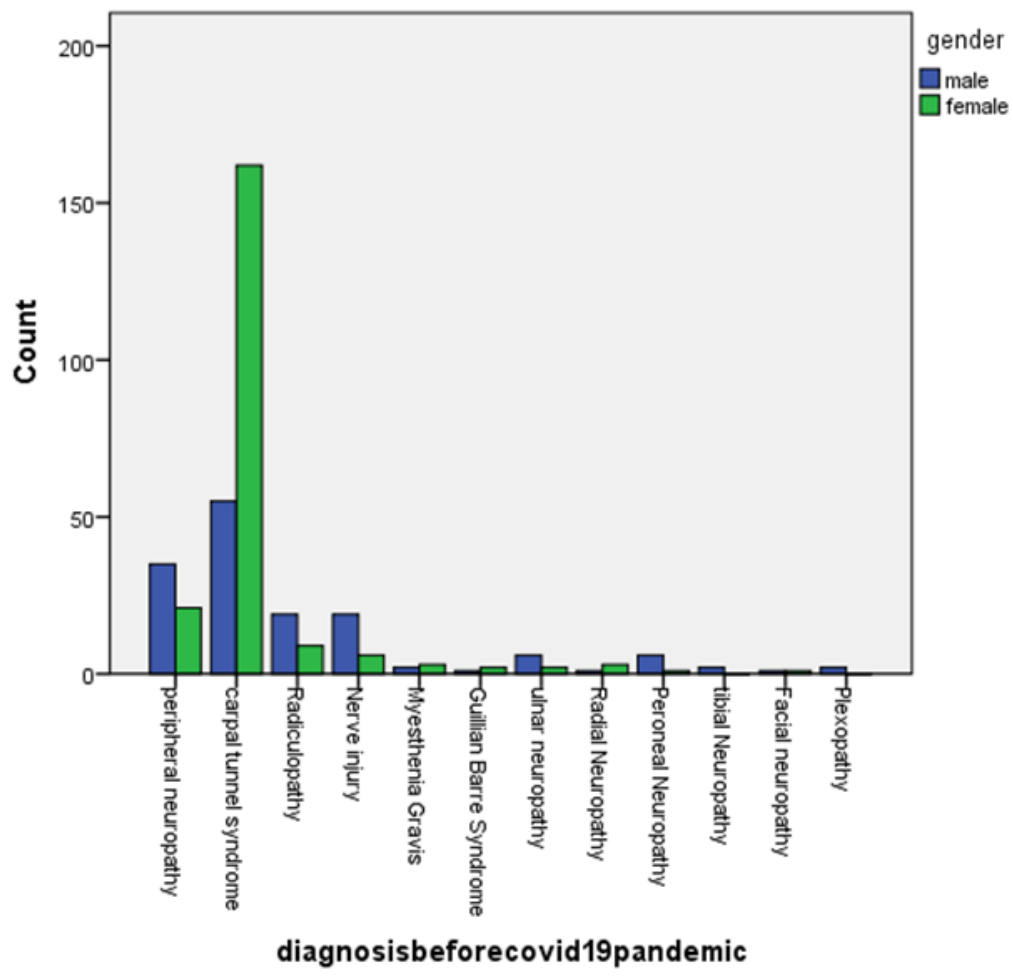

Figure 3 Distribution of neurological disorders and their frequencies according to gender before the Covid-19 pandemic

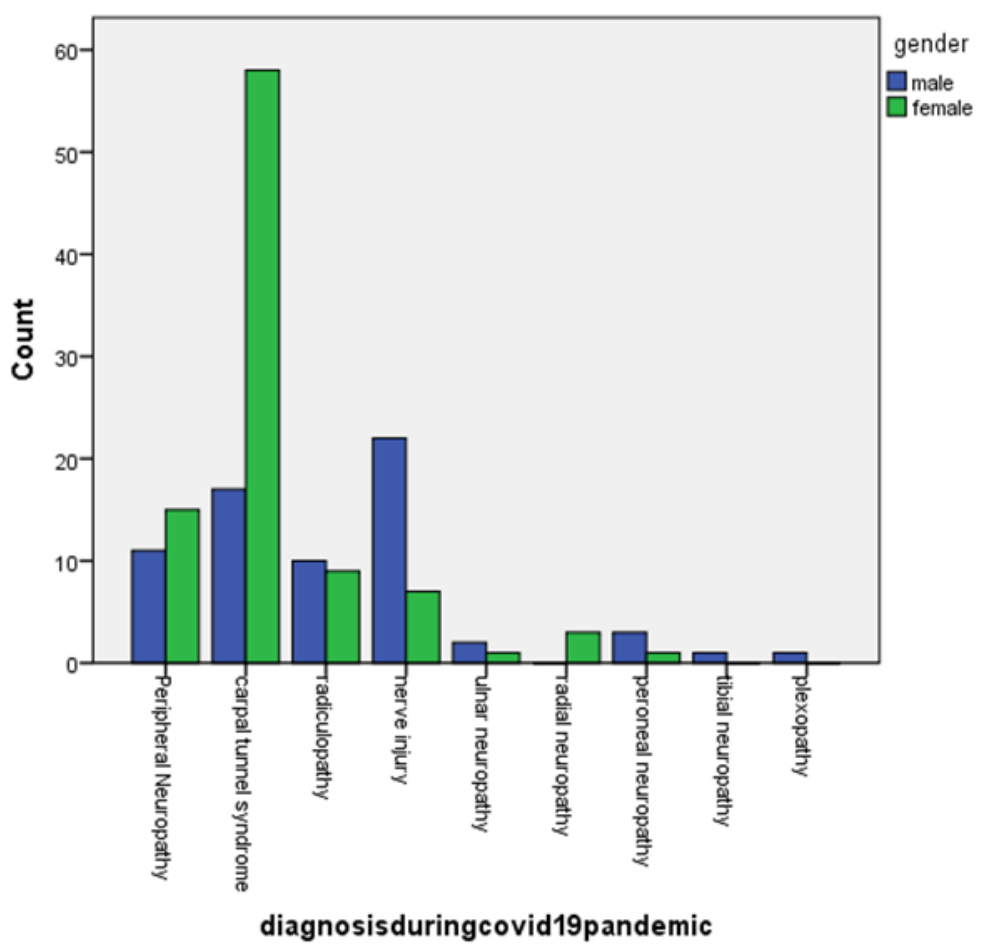

Figure 4 Distribution of neurological disorders and their frequencies according to gender during Covid-19 pandemic

Analysis of CTS patients showed the number of normal NCS in patients referred as CTS were about $40.05 \%$ during precovid period and has decreased to $28.57 \%$ during the Covid 19 pandemic similar to Egyptian study showing $30 \%$ normal NCS findings in referrals to EMG clinic for CTS[11]. The male to female ratio before Covid-19 pandemic was 1:2.95 with slightly higher female rate of 1:3.4 in the referrals during Covid-19 pandemic. An analysis of CTS diagnosis based on 
their severity was done. During the pre-covid studies $36.11 \%$ showed mild CTS, $50.56 \%$ moderate and $13.33 \%$ as severe CTS. In comparison to it, the profile of patients during the Covid- 19 pandemic era showed $26.2 \%$ mild CTS, $59.3 \%$ moderate and $14.49 \%$ severe CTS.

The analysis of nerve injury showed increased number of patients came with nerve injuries during the pandemic accounting for $18.01 \%$ compared to $6.96 \%$ before the covid 19 period. The male to female ratio of nerve injuries in EMG clinic was 1:0.32 in both the periods without any change.

The radiculopathies diagnosed in neurophysiological clinic has increased from $7.8 \%$ in pre-covid period to $11.8 \%$ during the pandemic. The percentage of peripheral neuropathies before covid and during pandemic were similar that is $15.6 \%$ and $16.1 \%$. But the male to female ratio had reversed with more females seeking attention with neuropathic pain symptoms during pandemic.

\section{Discussion}

The patients referred to EMG clinic in Al Noor Specialist Hospital are mostly from departments of Neurosurgery, Neurology, Orthopedics, Plastic surgery, Internal medicine, Nephrology and Oncology. It also receives patients from other hospitals in and around Makkah. These patients are referred according to clinical symptoms for further evaluation and confirmation of diagnosis, severity and type of neurological disorder. In our study we have found the physicians have become more fastidious in analyzing the clinical finding and symptoms during the pandemic observed as decline in number of referred patients showing normal study (46.58\% from $60.45 \%$ ). This is good in one way but may lead to missing out of mild cases and subclinical cases which would affect the prognosis and management adversely. Interestingly the number of traumatic nerve injuries referred to EMG clinic had increased from $6 \%$ pre-covid to $18 \%$ during pandemic. These people are mostly victims of car accidents and a few having occupational injuries or some selfinflicted wounds. Covid-19 pandemic has been very stressful all over the world with heightened stress, anxiety and depression. [12,13]. The percentage of patients showing neurophysiological findings of radiculopathies has increased from $7.8 \%$ during pre-covid to $11.8 \%$ during the pandemic. This might be attributed to prolonged sitting for hours with work from home during lockdowns $[14,15]$. People had been in bad posture with smartphones and laptops, many without proper home office setup with desk and chair showing symptoms of neck and back pain. These can be prevented by increasing awareness and teaching people about the importance of exercise, good posture, physically active, stretching exercises and healthy eating during lockdowns [16].

\section{Conclusion}

Covid- 19 was very stressful and disturbing to the normal functions of the population. It has its greatest toll on healthcare system. Many non-urgent consultations and treatments had taken a backseat which would definitely affect their prognosis and management. this study has highlighted the effect of pandemic on likelihood of missing milder diseases and subclinical diseases. It has also brought into attention the increased number nerve injuries during pandemic which should be cause of concern and also slight increase in radiculopathies has been seen may be as a consequence prolonged stay at home and work from home orders. Necessary steps should be taken to teach individuals to about stress management, importance physical activity and maintenance of good posture. The hospitals and healthcare system should be more prepared in future in such situations to maintain timely high-quality care for early detection of cases and not to miss the milder cases.

\section{Compliance with ethical standards}

\section{Acknowledgments}

We would like to thank the Staff of Neurophysiology Clinic Al Noor Specialist Hospital, Makkah Saudi Arabia for their valuable support in completing this project.

\section{Disclosure of conflict of interest}

The authors declare that there is no conflict of interest and no financial aid was taken for this project. 


\section{References}

[1] Brazier MA. The emergence of electrophysiology as an aid to neurology.in: Aminoff M.J. Electrodiagnosis in clinical neurology. 6th ed. Churchill Livingstone, Philadelphia. 2012; 7-8.

[2] Zhang M. Sheng Li Ke Xue Jin Zhan. 2013; 44(2): 158-160.

[3] Kazamel M, Warren P. History of electromyography and nerve conduction studies: A tribute to the founding fathers. Journal of Clinical Neuroscience. 2017; 43: 54-60.

[4] San-Juan D, Jiménez CR, Camilli CX, et al. Guidance for clinical neurophysiology examination throughout the COVID-19 pandemic. Latin American chapter of the IFCN task force - COVID-19. Clin Neurophysiol. 2020; 131(7): 1589-1598.

[5] WHO Director-General's opening remarks at the media briefing on COVID-19 - 3 March 2020 [Internet]. Who.int. 2021 [cited 19 March 2021].

[6] CSSEGISandData/COVID-19 [Internet]. GitHub. 2021 [cited 19 March 2021].

[7] Rubin D, Daube J. n.d. Clinical neurophysiology. 3rd ed. New York: Oxford university press. 802.

[8] Kassardjian C, Desai U, Narayanaswami P. Practical guidance for managing electromyography requests and testing during the COVID -19 pandemic. Muscle \& Nerve. 2020; 2(1): 30-33.

[9] Appendino JP, Baker SK, Chapman KM, et al. Practice Guidelines for Canadian Neurophysiology Laboratories During the COVID-19 Pandemic. Canadian Journal of Neurological Sciences / Journal Canadien des Sciences Neurologiques. 2021;48(1):25-30.

[10] San-Juan D, Jiménez CR, Camilli CX, et al. Guidance for clinical neurophysiology examination throughout the COVID-19 pandemic. Latin American chapter of the IFCN task force - COVID-19. Clin Neurophysiol. 2020; 131(7): 1589-1598.

[11] Abdel Kader A, Basheer M, Maher E, Elkholy S. Carpal tunnel syndrome among other referral diagnoses in the Egyptian Clinical Neurophysiology Unit. The Egyptian Journal of Neurology, Psychiatry and Neurosurgery. 2015; 52(3): 183.

[12] Khademian F, Delavari S, Koohjani Z, Khademian Z. An investigation of depression, anxiety, and stress and its relating factors during COVID-19 pandemic in Iran. BMC Public Health. 2021; 21(1).

[13] Shevlin M, McBride O, Murphy J, Miller J, Hartman T, Levita L et al. Anxiety, depression, traumatic stress and COVID-19-related anxiety in the UK general population during the COVID-19 pandemic. BJPsych Open. 2020; 6(6).

[14] A Side Effect of Working from Home: Back or Neck Pain That Needs Medical Attention | Resource | Baptist Health South Florida [Internet]. Resource | Baptist Health South Florida. 2021 [cited 20 March 2021].

[15] Qureshi. Neck pain problems on a rise due to Work from Home during lockdown, say doctors [Internet]. India Today. 2021 [cited 20 March 2021].

[16] 6 Ways to Alleviate Lower Back Pain During Lockdown - COVID-19, Featured, Health Topics, Pain Management, Physical Rehabilitation - Hackensack Meridian Health [Internet]. Hackensack Meridian Health. 2021 [cited 20 March 2021]. 